\title{
Evoked potentials to gustatory stimulations in cases with unilateral facial nerve palsy
}

Masashi Wada ${ }^{1}$

Masaoki Wada ${ }^{2}$

\begin{abstract}
Evoked potentials to taste stimulations were discussed. New our technique of objective measurement of gustatory function by an evoked potential technique will be introduced. As a result, introduction of taste solution on the tongue innervated by facial nerve in normal subjects resulted in positive responses. Absence of taste solution did not resulted in the positive response. In case with unilateral; facial nerve palsy, introduction of taste solution on the affected side did not resulted in the positive responses.
\end{abstract}

Keywords: auditory, brain stem, evoked potentials, nerve net, neurology.

\footnotetext{
${ }^{1}$ WADA Ear, Nose and Throat clinic; Tokyo - AC - Japan. E-mail: elprimero2015@yahoo.co.jp

2 DOCTOR, OKI CLINIC, IBARAKI - Japan. E-mail: elprimero2010@yahoo.co.jp

Institution: WADA Ear, Nose and Throat clinic.

Send correspondence to:

Masashi Wada

E-mail: elprimero2015@yahoo.co.jp

Paper submitted to the ITJ-SGP (Publishing Management System) on October 21, 2013;

and accepted on January 22, 2014. cod. 146
} 


\section{INTRODUCTION}

Where as the other sense organs function with only one nerve, the transmission of the sensation of taste to the brain is achieved by a number of nerves, including the glossopharyngeal, intermediate and a small section of the vagal nerve. And the gustatory nerve fibers of the intermediate nerve pass through a nerve bundle consisting of the lingual, greater petrosal and the facial nerve. The taste of food is sent to the brain as gustatory information passing through a total of four nerves on each side, that is glossopharyngeal, facial, vagal and greater petrosal nerve ${ }^{1,2}$. Thus, the separate taste examination of each area of gustatory innervation is necessary in clinical practice in treating patients with taste disorders. For example, in the taste examination of patients with peripheral facial nerve palsy such as Bell's palsy, the use of filter paper discs or electrogustometer renders possible detection of unknown taste disorder in more than $30 \%$ of the affected cases $^{1}$. But, these techniques have the disadvantage of being subjective.

Therefore, the separate taste examination of each area of gustatory innervation by an objective measurement of gustatory function such as evoked potential technique is expected to become the standard measuring technique. Many different approaches for the recording of gustatory evoked potential have been employed ${ }^{3-6}$. But as a result, none of them has been standardized for use in the clinical testing of taste. Now our technique of gustatory stimulations on the region of tongue innervated by the chorda tympani nerve is introduced to establish the objective measurement of gustatory function and to localize the facial nerve lesion by an evoked potential technique.

\section{MATERIAL AND METHODS}

The study population consisted of 11 apparently healthy Japanese male non-smokers 28-34 years. Our device for the recording of evoked response to taste stimulations is shown in Figure $1^{7,8}$.

The tip of stimulator is pressed onto the tongue so that the Bottle containing the taste solution pushes a switch attached to the bottom of the stimulator until the trigger pulses are generated by a switch.

An amount of taste solution (approximately $0.1 \mathrm{ml}$ ) is given in each presentation on the tongue through the polyvinyl formal sponge attached to the tip of stimulator. The polyvinyl formal sponge used is originally hard but soon become soon soft and elastic when wet. It will quickly expand again after administrating a taste solution. The tracing starts $300 \mathrm{~ms}$ prior to a trigger signal so that the switch is pressed just after a start of administrating taste solution on the tongue surface.

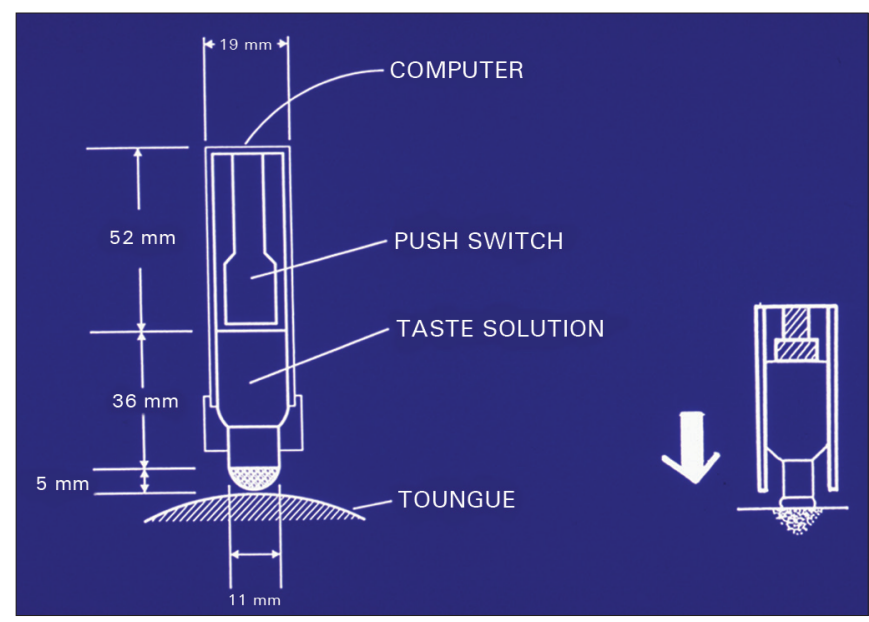

Figure 1. Device. Our device for recording evoked responses to taste stimulations. The tip of the stimulator is pressed on the tongue until a switch at the bottom of the stimulator is pushed by the bottle containing taste solution to generate a trigger pulse $\mathrm{s}^{7,8}$.

The most effective pressure to generate a trigger pulse in a certain period just after a start of administration of taste solution was $170 \mathrm{~g}$ according to our previous study. An electroencephalogram was recorded by the same method with olfactory evoked potentials. Practical technique of our examination is as follows; the subject is instructed to lie supine on a bed. The patient's tongue was protruded forward slightly, and experimenter held the apex of the tongue with a piece of gauze. The tip of stimulator is pressed vertically onto one side of the tongue until the trigger pulses are generated by switch attached to the bottom of the stimulator. After administrating taste solution, a subject's tongue surface is cleaned by using a filter paper gently and quickly. After eight responses had been recorded, the results were averaged by a "Neuropack 4 computer". Artificial saliva having no taste was applied as a control solution (Table 1). Each individual was tested mainly using two taste qualities, that is $20 \%$ Glucose and $10 \%$ sodium chloride.

Gustatory stimulation was presented once in 30 $\mathrm{s}$, so that the results were not affected by the fatigue of gustatory sense or adaptation. Each new and higher concentration of taste was presented after by an interval of $15 \mathrm{~min}$. The taste solutions and control solution given were maintained to be $30-34{ }^{\circ} \mathrm{C}$ to remove the effect of warm sensation. Because the solution of taste qualities show the maximum sensitivity near body temperature in the measurement of evoked potentials. The site of stimulation was the region of tongue innervated by the chorda tympani nerve. According to Tomita et al. ${ }^{1}$ the chorda tympani on the tongue exists at the apex of tongue and the left and right sides of chorda tympani nerves make a fan shaped crossing. Hence the site of stimulation in our experiment was $20 \mathrm{~mm}$ from the apex of tongue and $15 \mathrm{~mm}$ lateral side from center line. 
Table 1. Contents of Artificial saliva*

\begin{tabular}{lcc}
\hline \multicolumn{1}{c}{ Substance } & Contains $(\mathrm{mg} / 50 \mathrm{~g})$ & Concentration $(\mathrm{M} / \mathrm{l})$ \\
\hline Soduim $(\mathrm{NaCl})$ & 42.2 & $1.4 \times 10^{-2}$ \\
Potassium chloride $(\mathrm{KCl})$ & 60.0 & $1.6 \times 10^{-2}$ \\
Calcium chloride $\left(\mathrm{CaCl}_{2}\right)$ & 7.3 & $1.3 \times 10^{-3}$ \\
Magnesium chloride $\left(\mathrm{MgCl}_{2}\right)$ & 17.1 & $5.5 \times 10^{-4}$ \\
Monobasic potassium phosphate $\left(\mathrm{K}_{2} \mathrm{HPO}_{4}\right)$ & 17.1 & $2.0 \times 10^{-3}$ \\
\hline
\end{tabular}

Contents of artificial saliva; * Commercial name: Salivet ${ }^{\circledR}$ (Teijin. Co.; Ltda., Japan)

\section{RESULTS}

We detected no response in the absence of taste by the stimulation with Artificial Saliva in normal young male subjects (Figure 2).

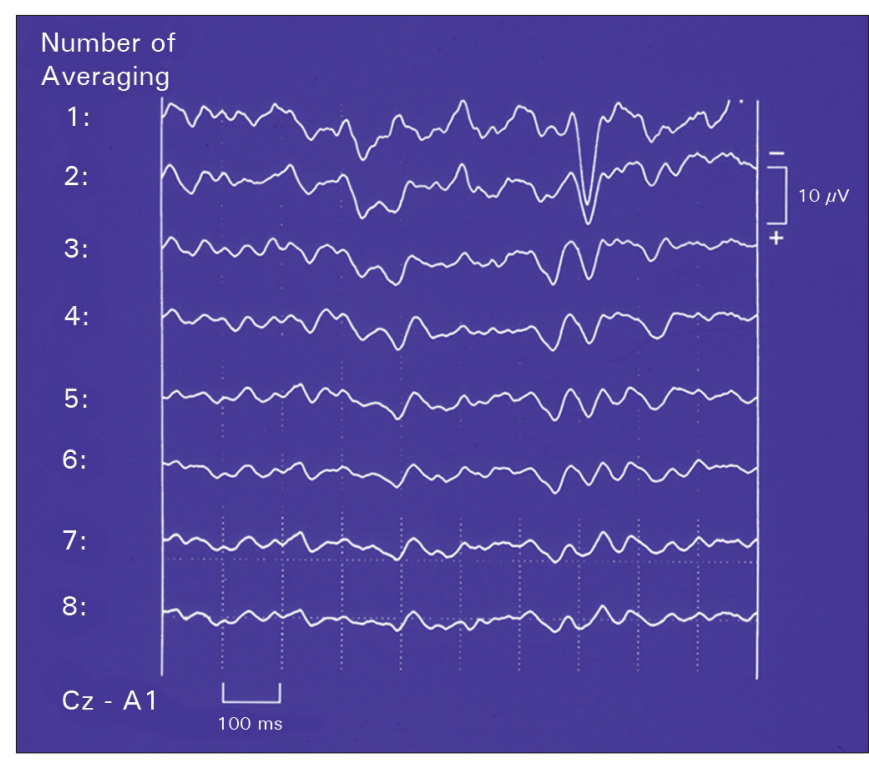

Figure 2. Response in the absence of taste. We detected no response in the absence of taste in normal young male subjects.

A typical pattern of evoked potentials to $20 \%$ glucose in normal young subject before averaging is shown here. Positive responses were detectable at a certain peak latency by using a superimposition technique (Figure 3 ). After averaging a positive response became obvious (Figure 4). In this graph, wave 8 represents 8 times the total. As seen in the figure, the following data were obtained. The wave 4 or 5 shows the largest amplitude and wave 7 or 8 represented the clearest wave. Administration of $20 \%$ evoked a positive response with a peak latency at $72-197 \mathrm{msec}$ in our 11 normal young subjects. A typical pattern of evoked potentials to $3 \%$ sodium chloride in a normal young subject before averaging is shown in Figure 5, and positive responses were detectable at a certain peak latency by using a superimposition technique (Figure 5). After averaging, a positive response became obvious (Figure 6). Also, each new and higher concentration of taste solution

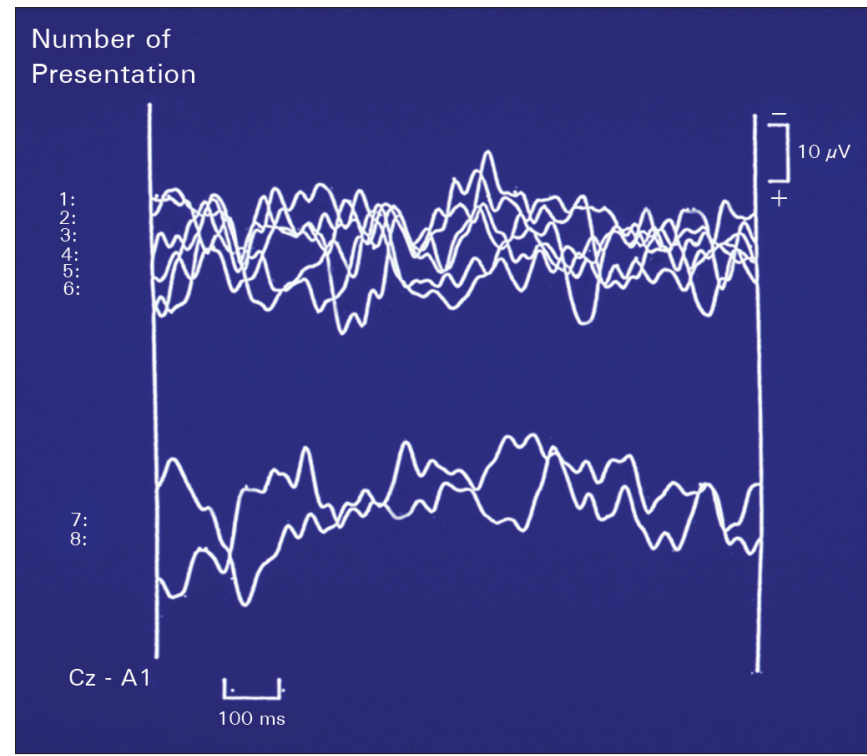

Figure 3. Response to $20 \%$ glucose. A typical pattern of an evoked potential to $20 \%$ glucose in normal young subject before averaging is shown here. Positive responses were detectable at a certain peak latency by using a superimposition technique.

was presented after an interval of 15 minutes. Administration of $10 \%$ sodium chloride revealed a positive response with a shorter peak latency, as is shown in Figure 7. The shortening of peak latency was recorded for the more concentrated taste solution. Administration of $10 \%$ sodium chloride evoked a positive response with a peak latency at $84 \mathrm{~ms}$ to $188 \mathrm{~ms}$ in these 11 healthy young male subjects.

In our study, the positive waves were distinguishable by using the technique of superimposition before averaging, and the positive wave was rendered clearer by averaging. Also, no detectable response was seen in the absence of taste in normal subjects.

Accordingly, the positive response to the taste solution with a peak latency at around 70 to $200 \mathrm{~ms}$ in our result can be considered a specific result of gustatory stimulation. However, the latency of positive response to the taste solution presented here has spread over the wide range of about $70-200 \mathrm{~ms}$. This was mainly due to the difference in elasticity or softness of the tongue. This peak latency of positive response obtained by our method may be not a true latency associated with the 


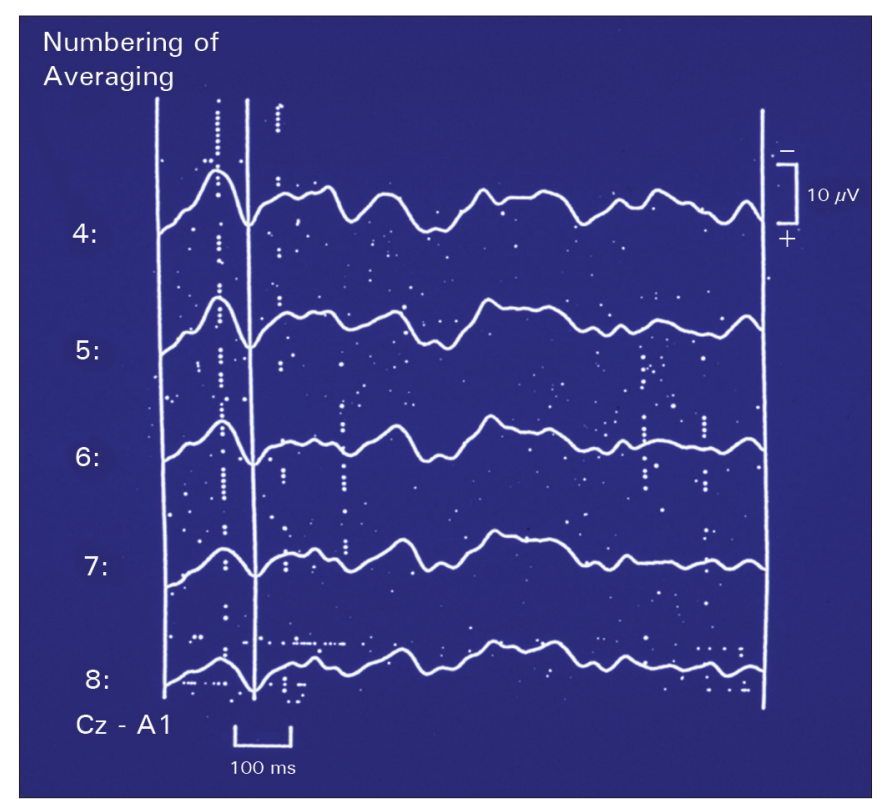

Figure 4. Response to $20 \%$ glucose. After averaging a positive response became obvious. In this graph, wave 8 represents 8 times the total. As seen in the figure, the following data were obtained. The wave 4 or 5 shows the largest amplitude and wave 7 or 8 represented the clearest wave. Administration of $20 \%$ evoked a positive response with a peak latency at $72-197 \mathrm{msec}$ in our 11 normal young subjects.

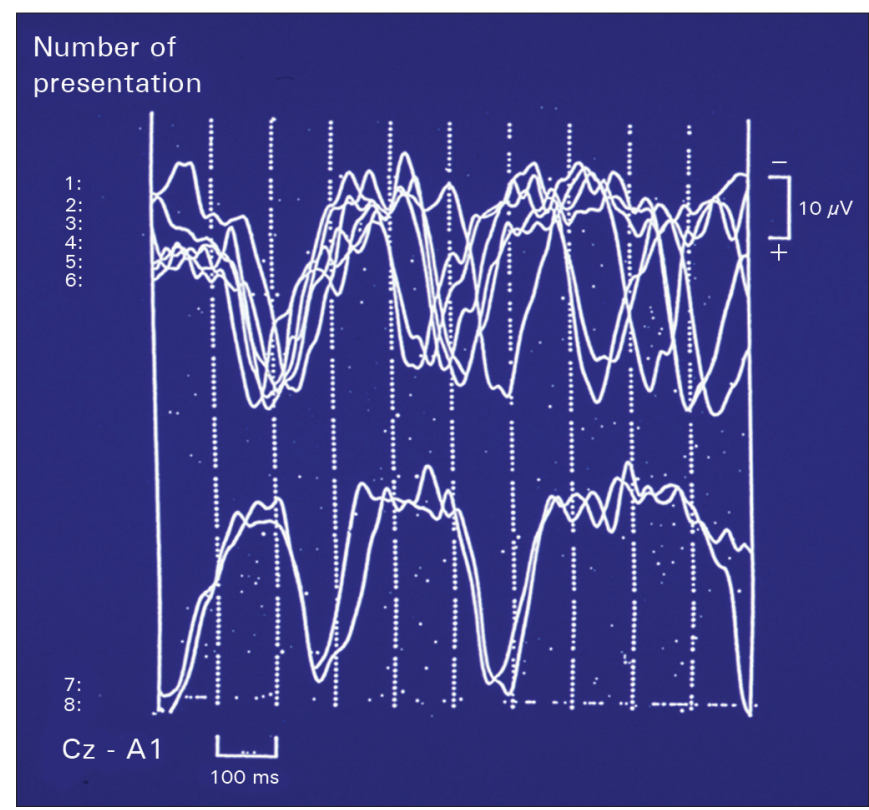

Figure 5. A typical pattern of evoked potentials to $3 \%$ sodium chloride in a normal young subject before averaging. Positive responses were detectable at a certain peak latency by using a superimposition technique.

taste stimulation. Because a time lag exists for the dose of taste solution, that is, there existed the time difference between the touching time of taste solutions on the tongue and a generating time of trigger signal. In our method, the tracing starts $300 \mathrm{~ms}$ prior to a trigger

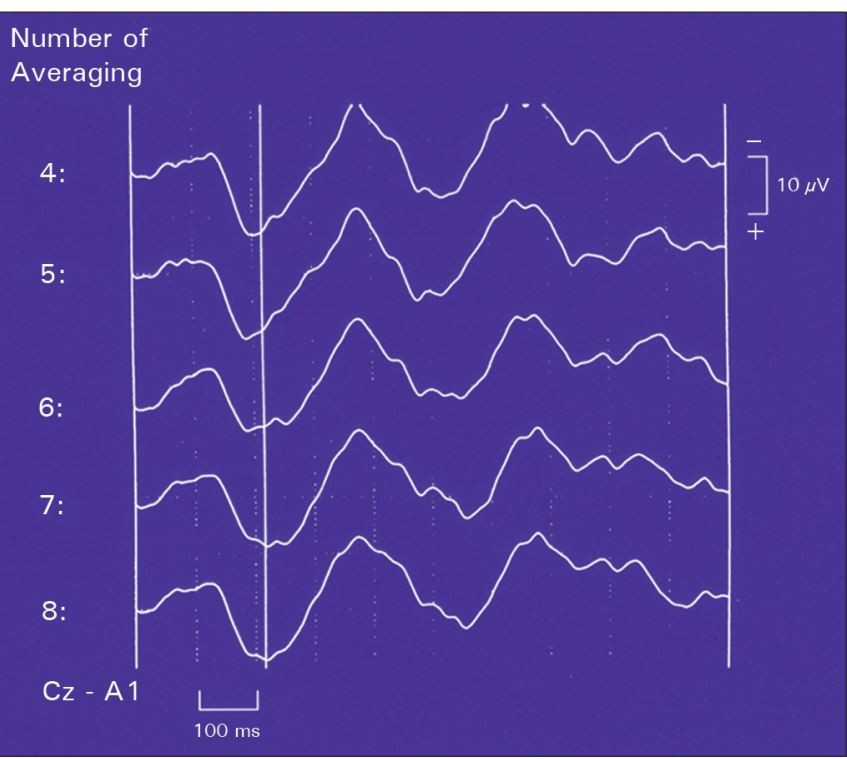

Figure 6. Response to $3 \%$ sodium chloride. After averaging, a positive response became obvious.

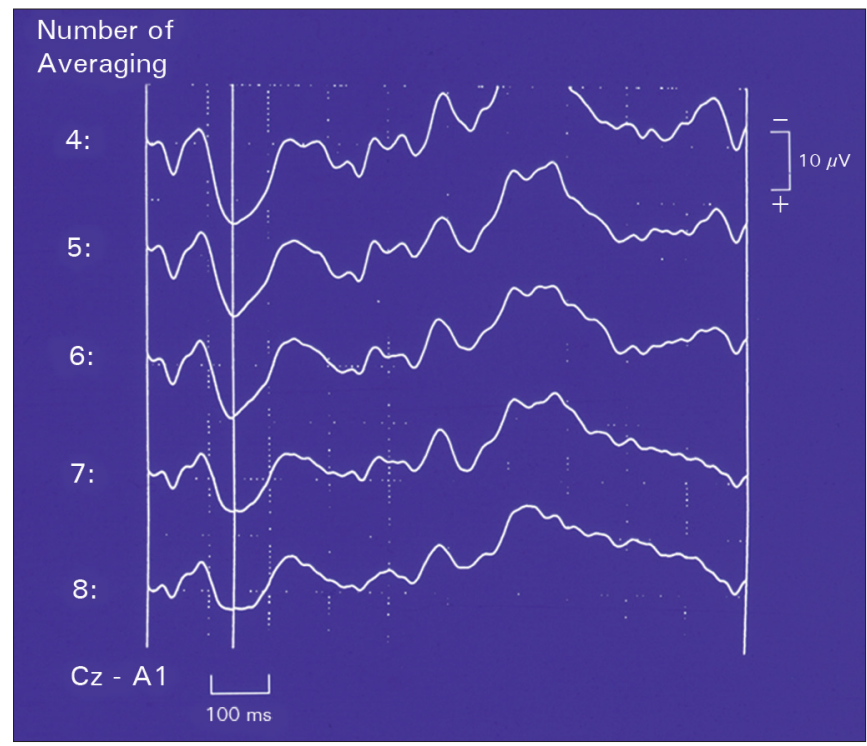

Figure 7. Response to $10 \%$ sodium chloride. Administration of $10 \%$ sodium chloride revealed a positive response with a shorter peak latency. The shortening of peak latency was recorded for the more concentrated taste solution.

signal so that the switch for the trigger signal should be pressed just after a strat of administrating a taste solution on the tongue.

Next, the difference in the response between the ipsilateral and colateral response were discussed (Figure 8). As is shown here, ipsilateral response by the recording from the same side with the taste stimulation may have a tendency to show the larger amplitude of main positive response with a shorter peak latency compare to the contralateral response by the recording from the opposite side with the taste stimulation. 


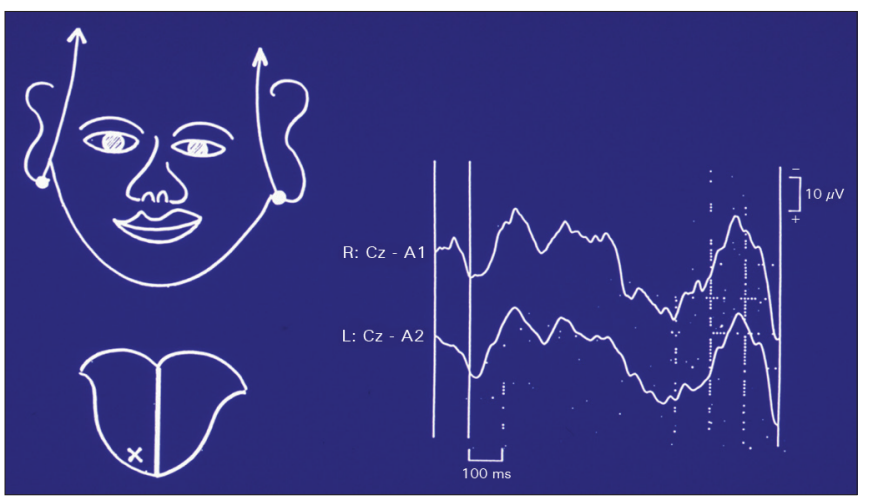

Figure 8. Ipsilateral response and contralateral response. The difference in the response between the ipsilateral and contralateral response were discussed. As is shown here, ipsilateral response by the recording from the same side with the taste stimulation may have a tendency to show the larger amplitude of main positive response with a shorter peak latency compare to the contralateral response by the recording from the opposite side with the taste stimulation.

Next, responses in case with facial palsy were discussed.

Administration of $20 \%$ glucose on the affected side of tongue as that of the lesion did not evoke a positive response (Figure 9).

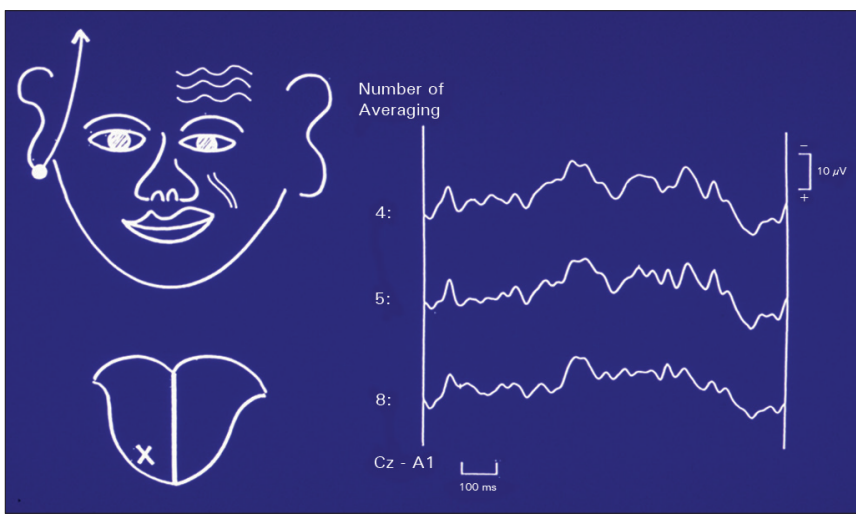

Figure 9. Response in case with facial nerve palsy. Administration of $20 \%$ glucose on the affected side of tongue as that of the lesion did not evoke a positive response.

On the other hand, positively evoked potentials were recorded by the administration of $20 \%$ glucose on the opposite side of the tongue as that of the lesion (Figure 10).

Next, the bilateral recording in case with unilateral facial nerve palsy was discussed. First, the evoked potentials by the stimulation on the same side of the tongue as that of the lesion were studied. As is shown here, positively evoked potentials were not recognized in both recording from same side and opposite side with a stimulation (Figure 11). In this graph, $\mathrm{R}$ represents a

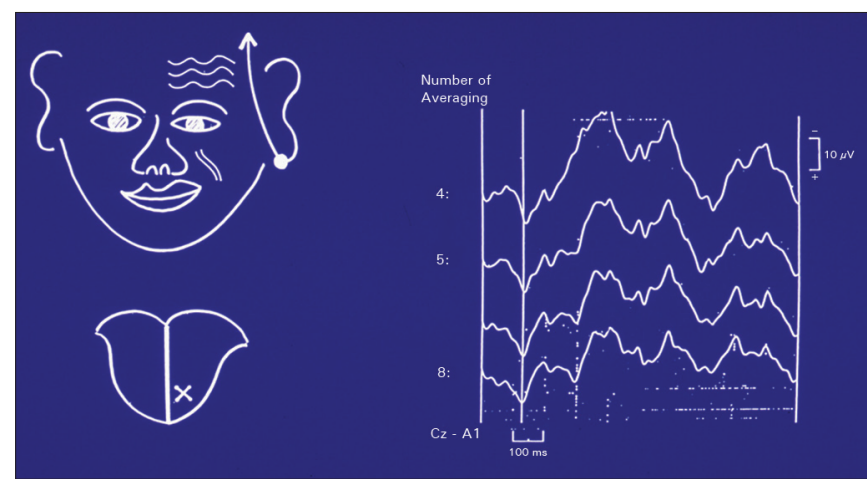

Figure 10. Response in case with facial nerve palsy. On the other hand, positively evoked potentials were recorded by the administration of $20 \%$ glucose on the opposite side of the tongue as that of the lesion.

result by the recording from the right side, affected side in this case and $L$ represents a result by the recording from right side, affected side. Top 2 tracings represents 4 times the total, middle 2 tracings represents 8 times the total and the lowest 2 tracings represents 12 times the total.

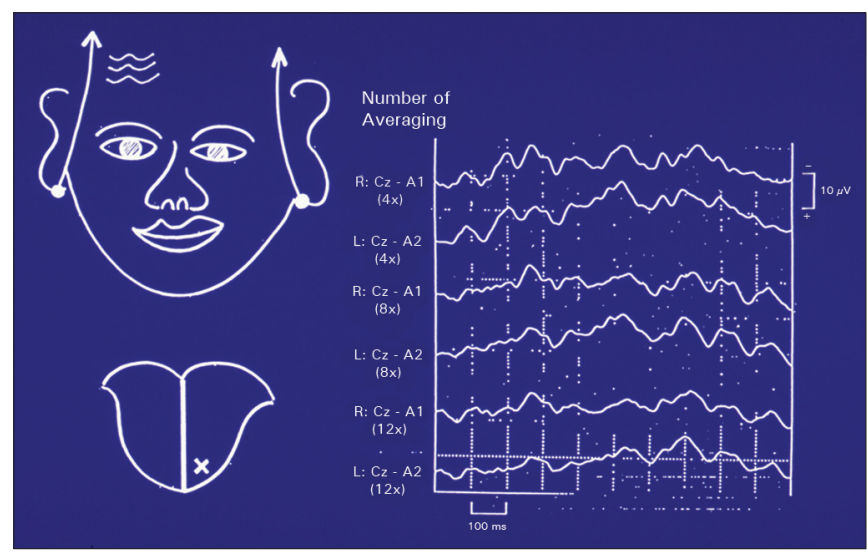

Figure 11. Evoked potentials by the recording from both sides. The bilateral recording in case with unilateral facial nerve palsy was discussed. First, the evoked potentials by the stimulation on the same side of the tongue as that of the lesion were studied. As is shown here, positively evoked potentials were not recognized in both recording from same side and opposite side with a stimulation. In this graph, $R$ represents a result by the recording from the right side, affected side in this case and $L$ represents a result by the recording from right side, affected side. Top 2 tracings represents 4 times the total, middle 2 tracings represents 8 times the total and the lowest 2 tracings represents 12 times the total.

On the other hand, administration of $10 \%$ sodium chloride on the unaffected side of the tongue as that of the lesion resulted in detectable positive response (Figure 12). As is shown in this figure, positively evoked potentials were recognized in both recording, and larger amplitude of the main positive response was recognized in the recording from the same side with stimulation. 


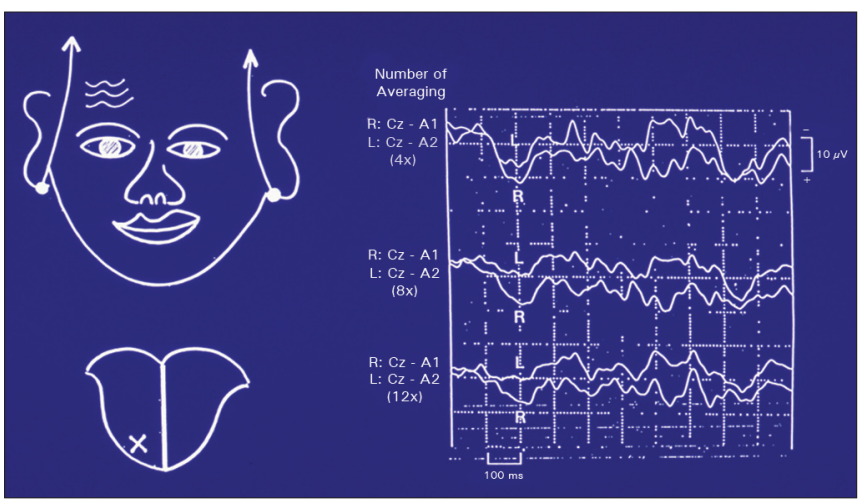

Figure 12. Evoked potentials by the recording from both sides. On the other hand, administration of $10 \%$ sodium chloride on the unaffected side of the tongue as that of the lesion resulted in detectable positive response. As is shown in this figure, positively evoked potentials were recognized in both recording, and larger amplitude of the main positive response was recognized in the recording from the same side with stimulation.

\section{DISCUSSION}

Recording of gustatory evoked potential for the objective evaluation of gustatory function was attempted by many investigators, but a method in clinical practice in testing patients with taste disorders has not been standardized yet. Gustatory evoked potentials were not easily elicited owing to problems associated with control of the stimulus.

One reason mentioned for this is the confusion of gustatory evoked potential includes many sensations not only taste but touch, pressure, and warmth 4 . Also, owing to chemical substances, quantifying the stimulus of a taste solution is difficult.

Moreover, the following basic problems should be considered when administrating a taste solution to subject's tongue. First, the timing of triggering for the sake of averaging of evoked potentials, the duration of stimulation, and the area of stimulation should be investigated. Second, in the addition of successive taste solution stimuli, the fatigue or the adaptation of tongue, by which the sensation for the taste solution decrease or completely disappears, occurs and a definite time lag is required for the recovery from these phenomena. After examining the foregoing problems, we formed our new apparatus stimulating taste solution to tongue.

The literature contains discussion of the evoked potentials to gustatory stimulations.

Funakoshi \& Kawamura reported firstly gustatory evoked potentials ${ }^{3}$. After that, some reports have been made by Kobal ${ }^{4}$, Maetani et al. ${ }^{5}$, Min \& Sakamoto ${ }^{9,10}$. $\mathrm{Kobal}^{4}$ developed the apparatus for gaseous taste stimulus to remove touch stimuli.

However, the stimulus did not make clear the definite innervation area of the taste organ achieved by the gaseous stimulus.
Min \& Sakamoto ${ }^{9,10}$ devised a new method. When the tip of the bottle of taste solution comes into contact with the area innervated by the chorda tympani nerve of the tongue, the taste solution is placed on the tongue and at the same time laser beam detects the touching moment of the tip of stimulator without contact between the device and the tongue. However, this technique is not available for use in the clinical testing of taste.

According to our technique for the recording of gustatory evoked potentials, a tip of stimulator is pressed vertically onto one side of the tongue until the trigger pulses are generated by switch attached to the bottom of the stimulator.

The site of stimulation was the region of tongue innervated by the chorda tympani nerve. According to our results, the positive waves were distinguishable by using the technique of superimposition before averaging, and the positive wave was rendered clearer by averaging

Also, no detectable response was obtained in the absence of taste in normal young subject.

Thus, the positive responses with a peak latency of 70 to $200 \mathrm{~ms}$ are evoked by the taste solutions (as described in our technique) and not by some extraneous stimuli associated with taste presentation (e.g.; reaction to the tactile sense of the solution, a press of simulator on the tongue surface, holding of the tongue apex).

This evoked potential technique will be useful for the objective evaluation of gustatory function of facial nerves, for detecting the presence of taste disorders, and for making an early diagnosis of facial nerve disorders.

\section{REFERENCES}

1. Tomita $\mathrm{H}$, Ikeda M, Okuda $\mathrm{Y}$. Basis and practice of clinical taste examinations. Auris Nasus Larynx. 1986;13 Suppl 1:S1-15.

2. Defour JJ. Facial Nerve Function and Assesment. In: Alberti PW, Ruben RJ (eds). Otologic Medicine and Surgery. Vol 1. New York: Churchill Livingstone; 1988. p.523-37.

3. Funakoshi M, Kawamura Y. Summated cerebral evoked responses to taste stimuli in man. Electroencephalogr Clin Neurophysiol. 1971;30(3):205-9. DOI: http://dx.doi.org/10.1016/00134694(71)90055-1

4. Kobal G. Gustatory evoked potentials in man. Electroencephalogr Clin Neurophysiol. 1985;62(6):449-54. PMID: 2415341 DOI: http:// dx.doi.org/10.1016/0168-5597(85)90055-3

5. Maetani C, Notake N, Takemoto I, Koizuka I, Ogino H, Matsunaga T, et al. Gustatory evoked potentials by taste solution. Chem Senses. 1989;14(2):311.

6. Ikui A. Gustatory evoked potentials by stimulation with $\mathrm{NaCl}$ solution in man (in Japanese). J Nihon Univ Med Ass. 1998;47(3):363-73.

7. Wada M. Evoked Response to Gustatory Stimulations (in Japanese). Jpn Taste Smell Res. 1995;2(3):185-8.

8. Wada M. Evoked response to taste stimulations. Int Tinnitus J. 2005;11(1):43-7.

9. Min BC, Sakamoto K. An analysis of gustatory evoked potentials for the four qualities with a special reference in individual taste thresholds (in Japanese). Jpn Taste Smell Res. 1997;4(1):43-54.

10. Min BC, Sakamoto K. Influence of sweet suppressing agent on gustatory brain evoked potentials generated by taste stimuli. App Human Sci. 1998;17(1):9-17. PMID: 9575639 DOI: http://dx.doi. org/10.2114/jpa.17.9 